\title{
Glabrous Rice 1, encoding a homeodomain protein, regulates trichome development in rice
}

\author{
Jinjun $\mathrm{Li}^{1 \dagger}$, Yundong Yuan ${ }^{2+}$, Zefu Lu², Liusha Yang ${ }^{2}$, Rongcun Gao ${ }^{1}$, Jingen Lu', Jiayang $\mathrm{Li}^{2}$ and Guosheng Xiong ${ }^{2^{*}}$
}

\begin{abstract}
Background: Glabrous rice, which lacks trichomes on the rice epidermis, is regarded as an important germplasm resource in rice breeding. Trichomes are derived from aerial epidermal cells and used as a model to study the cell fate determination in plant. In Arabidopsis, the molecular mechanisms of trichome development have been well studied. However, little is known about the molecular basis of trichome development in rice.

Results: In this study, near isogenic lines harboring the glabrous rice 1 locus were developed. By a map-based approach, we narrowed down the locus to a 21-kb DNA region harboring two genes. One of the genes named Glabrous Rice 1 (GLR1), which is most likely the candidate, encodes a homeodomain protein containing the WOX motif. Constitutive Expression of GLR1 could partially complement the glabrous phenotype of $\mathrm{NL}^{\text {glr }}$. The knock down of GLR1 by RNA interference led to a significant decrease in trichome number on the leaves and glumes of the RNAi transgenic plants.
\end{abstract}

Conclusion: GLR1 plays an important role in rice trichome development and will contribute to breeding of glabrous elite rice varieties.

Keywords: Glabrous rice, Trichome development, WOX protein

\section{Background}

The glabrous feature of rice is considered as a favorite agronomic trait for rice farmers because it has greater packing capability of rice grains and produces less dust that causes itching effect on farmers. Glabrous rice lacks trichomes on leaves and glumes (Khush, et al. 2001). Most rice cultivars in America are glabrous and recognized as an important germplasm resource in breeding due to its high yield, good quality, and wide compatibility in crossing with other rice varieties (Guo et al. 1999, Luo et al. 2000). Trichomes are derived from aerial epidermal cells and serve various protective purposes such as insect herbivore resistance, freezing tolerance, and shade of UV irradiation (Ishida et al. 2008). There are two distinct types of trichomes developed on leaves of monocot plants. One is macrohairs on silica cells, the other is microhairs along the stomata cells (Khush, et al. 2001). So far, a number of

\footnotetext{
* Correspondence: gsxiong@genetics.ac.cn

${ }^{\dagger}$ Equal contributors

${ }^{2}$ State Key Laboratory of Plant Genomics and National Center for Plant Gene Research, Institute of Genetics and Developmental Biology, Chinese

Academy of Sciences, Beijing 100101, China

Full list of author information is available at the end of the article
}

glabrous mutants have been identified in many plant species, including Arabidopsis, tomato, cotton, and maize (Machado et al. 2009, Moose et al. 2004, Rerie et al. 1994, Yang et al. 2011). However, the molecular mechanism underlying trichome development has only been intensively investigated in Arabidopsis.

In Arabidopsis, trichome development has been used as a model system to study the cell fate determination and shown to be regulated by a complex gene network (Ishida et al. 2008). A homeodomain-leucine zipper protein GLABRA2 (GL2) and an R3 Myb protein TRIPTYCHON (TRY) play essential roles in trichome initiation and hairless cell differentiation (Rerie et al. 1994, Schellmann et al. 2002). The expression of GL2 and TRY are regulated by the WD-repeat/bHLH/MYB complex including TRANSPARENT TESTA GLABRA1 (TTG1), GLABRA3 (GL3)/ENHANCER OF GLABRA3 (EGL3) and GLABRA1 (GL1). Epidermal cells expressing the GL2 protein are able to differentiate into trichome cells. The TRY protein expressed in trichome cells, however, can move into neighboring cells and compete with GL1 for binding to GL3/EGL3 to repress the GL2 expression. The TRY mediated down regulation of the GL2

\section{SpringerOpen ${ }^{\circ}$}

(c) 2012 li et al.; licensee Springer. This is an Open Access article distributed under the terms of the Creative Commons Attribution License (http://creativecommons.org/licenses/by/2.0), which permits unrestricted use, distribution, and reproduction in any medium, provided the original work is properly cited. 
expression inhibits trichome formation in neighboring cells (Ishida, et al. 2008). Actually, factors able to modulate this gene network affect the trichome development. Previous studies on mutants defective in the biosynthesis and/or signaling of gibberellins, salicylic acid, jasmonic acid, and cytokinin have showed that phytohormones are involved in trichome initiation (Gan et al. 2006, Gan et al. 2007b, Perazza et al. 1998, Traw and Bergelson 2003, Zhou et al. 2011). It has been turned out that roles of these phytohormones in trichome development are mediated by their effect on the expression or activity of the components of the WD-repeat/bHLH/MYB complexes. Roles of gibberellins and cytokinins in trichome initiation are mainly dependent on $\mathrm{C} 2 \mathrm{H} 2$ transcription factors including GIS1, GIS2, ZFP5 and ZFP8. These transcription factors are able to promote the GL1 expression (Gan et al. 2007a, Maes et al. 2008, Perazza, et al. 1998, Zhou, et al. 2011). In addition, JAZ proteins, the key components in the JA signaling pathway, have been shown to interact with bHLH transcription factors (GL3, EGL3 and TT8) and MYB transcription factors (MYB75 and GL1) (Qi et al. 2011). The JA-induced destruction of JAZ proteins results in releasing the transcriptional function of the WD-repeat/bHLH/MYB complex and activating downstream events of trichome initiation. Furthermore, recent studies have shown that the microRNA156 targeted gene SPL9 could bypass the function of GL1 and directly binds to promoters of TCL1 and TRY to activate their expression ( $\mathrm{Yu}$ et al. 2010).

In contrast to the sophisticated mechanisms revealed in Arabidopsis, little is known about the molecular mechanisms of trichome development in other plants. It has been noted that a couple of homeodomain-leucine zipper proteins, which are specifically expressed in epidermal cells, are essential in differentiation of epidermal cells. Outer Cell Layer 4 (OCL4), a maize HD-ZIP transcription factor, has been suggested to involve in the repression of macrohair differentiation (Vernoud, et al. 2009), and a HD-Zip protein in tomato, Woolly (Wo) that interacts with Cyclin B2, plays an essential role for trichome formation and embryonic development (Yang et al. 2011). In addition, another subfamily of the homeobox gene, known as WUS-like homeobox genes $(W O X)$, may also play roles in division or differentiation of epidermal cells. Pressed Flower (PRS) is involved in activation of the proliferation of marginal cells. It has been observed that multicellular bulges with trichomes formed on stems and epidermal cells outgrow on sepals of 35S:PRS transgenic plants (Matsumoto and Okada 2001). Moreover, Narrow sheath 1 (NS1) and Narrow sheath 2 (NS2), which are duplicated relatives of PRS in maize, have been suggested to play a role in a lateral domain of shoot apical meristems (Nardmann et al. 2004).
In addition, OsWOX3 has been found to repress the expression of OsYAB3, which is required for cell differentiation during rice leaf development (Dai et al. 2007).

Previous study showed that macrohairs on the leaf blade are greatly reduced in the maize macrohairless 1 (mhl1) mutant (Moose, et al. 2004). A major QTL controlling macrohairs in Teosinte has been found to locate near the maize gene MHL1 (Lauter et al. 2004). In rice, previous genetic analysis has identified a couple of loci that control trichome development. For example, $g l$ regulates glabrous leaf and hull traits, $\mathrm{Hl}_{a}$ and $\mathrm{Hl}_{b}$ were related to long hair development on rice leaves and $\mathrm{Hg}$ may be responsible for the extreme long hairs on auricles and glumes (Nagao et al. 1960). However, no gene controlling these traits has been cloned in rice as yet. Recently, glabrous leaf and hull mutant (gl1) has been reported to locate within a 54-kb region at the short arm of chromosome 5 (Li et al. 2010, Wang et al. 2009, $\mathrm{Yu}$ et al. 1995), but the gene has not been identified yet. Here, we report the identification and characterization of the Glabrous Rice 1 (GLR1), which controls the trichome development in rice. Our work extends an insight into the molecular mechanism of trichome development in rice. The identification and characterization of GLR1 will facilitate breeders to develop elite glabrous rice varieties via marker-assisted-selection and genetic modification approaches.

\section{Results}

\section{Phenotype of the near isogenic line of glabrous rice}

The glabrous variety Jia64 is derived from the American rice variety Rico No.1 and near isogenic lines (NIL) of glabrous rice developed by backcrossing Jia64 with a pubescent variety Jia33 for 5 generations. There are no obvious differences of the overall morphology between $\mathrm{NIL}^{\text {GLRI }}$ and NIL ${ }^{g l r 1}$ plants (Figure 1a). However, the leaves of $\mathrm{NIL}^{\text {glrl }}$ plants are smooth whereas leaves of NIL ${ }^{\text {GLR1 }}$ plants are rough with many hairs. In contrast to glumes of the NIL ${ }^{\text {GLRI }}$ plant (Figure 1b), the glumes of the NIL ${ }^{g l r l}$ plant showed no trichome or only a few trichomes growing on margins of the hull (Figure 1c). On rice leaves, there are two types of trichomes, macrohairs and microhairs. Scan Electronic Microscope (SEM) analysis showed that both macrohairs and microhairs on the abaxial and adaxial sides of NIL ${ }^{G L R I}$ leaves are able to be observed (Figure 1d and Figure 1e). However, neither macrohairs nor microhairs could be observed on both sides of NIL ${ }^{g l r 1}$ leaves (Figure 1f and Figure 1g).

\section{Map-based cloning of GLR1}

Previous genetic analysis has shown that the glabrous phenotype of America rice was controlled by a single recessive nuclear gene (Li et al. 1993). To map the GLR1 locus, an $F_{2}$ mapping population was generated from a cross 

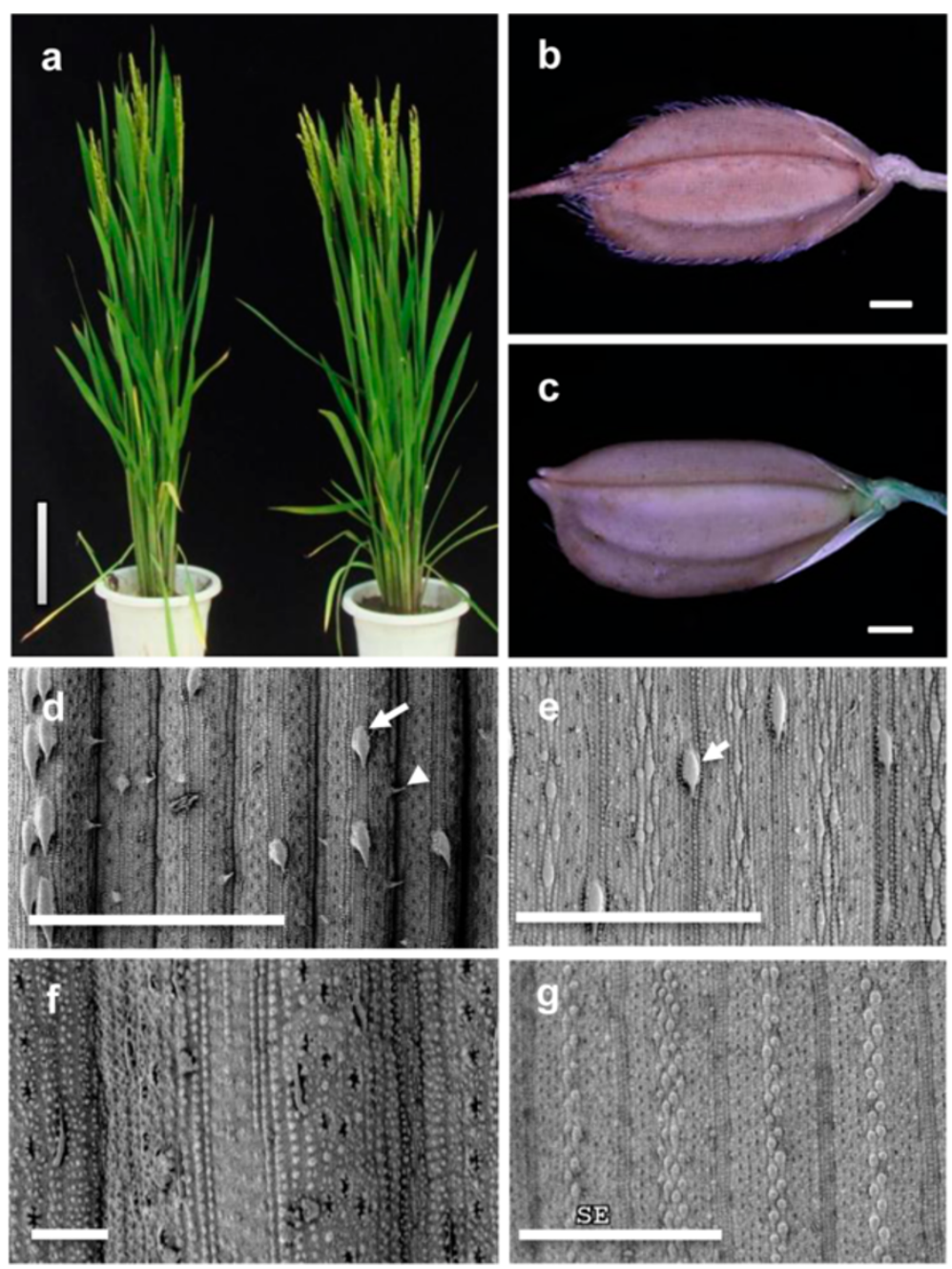

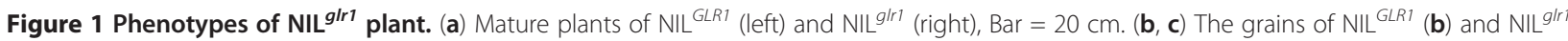
(c) Bars $=0.1 \mathrm{~cm}$. (d to $\mathbf{g}$ ) The SEM views of the adaxial (d) and abaxial (e) sides of the NIL ${ }^{G L R 1}$ leaves, and adaxial (f) and abaxial (g) sides of the $\mathrm{NIL}^{\text {glr } 1}$ leaves. Arrow indicates the macrohair and arrowhead shows the microhair. Bars $=500 \mu \mathrm{m}$.

between Jia64 and a polymorphic japonica variety Jia33. Linkage analysis of $44 \mathrm{~F}_{2}$ plants having the glabrous phenotype showed that the GLR1 locus located between the InDel marker M1 and the SSR marker M2 on chromosome 5 (Figure 2a and Table 1). This region is consistent with the previously mapped $g l 1$ locus on the short arm of chomsome 5 (Li, et al. 2010, Wang, et al. 2009). To fine-map GLR1, 1,447 $\mathrm{F}_{2}$ glabrous plants were analyzed using 7 newly developed markers (Figure $2 \mathrm{~b}$ and Table 1 ) and GLR1 was finally pin-pointed within an interval of 21-kb DNA fragment between the markers M6 and M7. Within this region, there are 2 predicted genes, LOC_Os05g02720 (Os05g0118600) and LOC_Os05g02730 (Os05g0118700) (Figure 2c). The former encodes a hypothetic protein and the latter encodes a homeobox-containing protein. Sequence analysis showed that LOC_Os05g02730 shares similarity to PRS in Arabidopsis, NS1 and NS2 in maize, and
OsWOX3 in rice (Dai, et al. 2007, Matsumoto and Okada 2001, Nardmann, et al. 2004). There are a conserved homeodomain at the $\mathrm{N}$ terminal and a conserved WOX motif at the $\mathrm{C}$ terminal of these proteins (Figure 3). Phylogenic analysis indicated that LOC_Os05g02730 belongs to a small NS/WOX3 subgroup consisting of OsWOX3, PRS, NS1 and NS2 (Dai, et al. 2007). We sequenced and compared the 21-kb DNA fragments between markers M6 and M7 from the NIL ${ }^{G L R 1}$ and NIL ${ }^{g l r 1}$. There is no difference in this region between NIL ${ }^{\text {GLR1 }}$ and NIL ${ }^{g l r 1}$ plants. To understand which gene, LOC_Os05g02720 or LOC_Os05g02730, is responsible for the phenotype, we analyzed their expression levels by RT-PCR. Compare to NIL ${ }^{G L R 1}$, the expression level of LOC_Os05g02720 decreased in the NIL ${ }^{g l r 1}$ plant (Figure 2d). However, the expression of LOC_Os05g02730 was dramatically reduced in the NIL ${ }^{g l r 1}$ plant (Figure 2d). The previous studies showed that the NS/WOX3 subgroup 

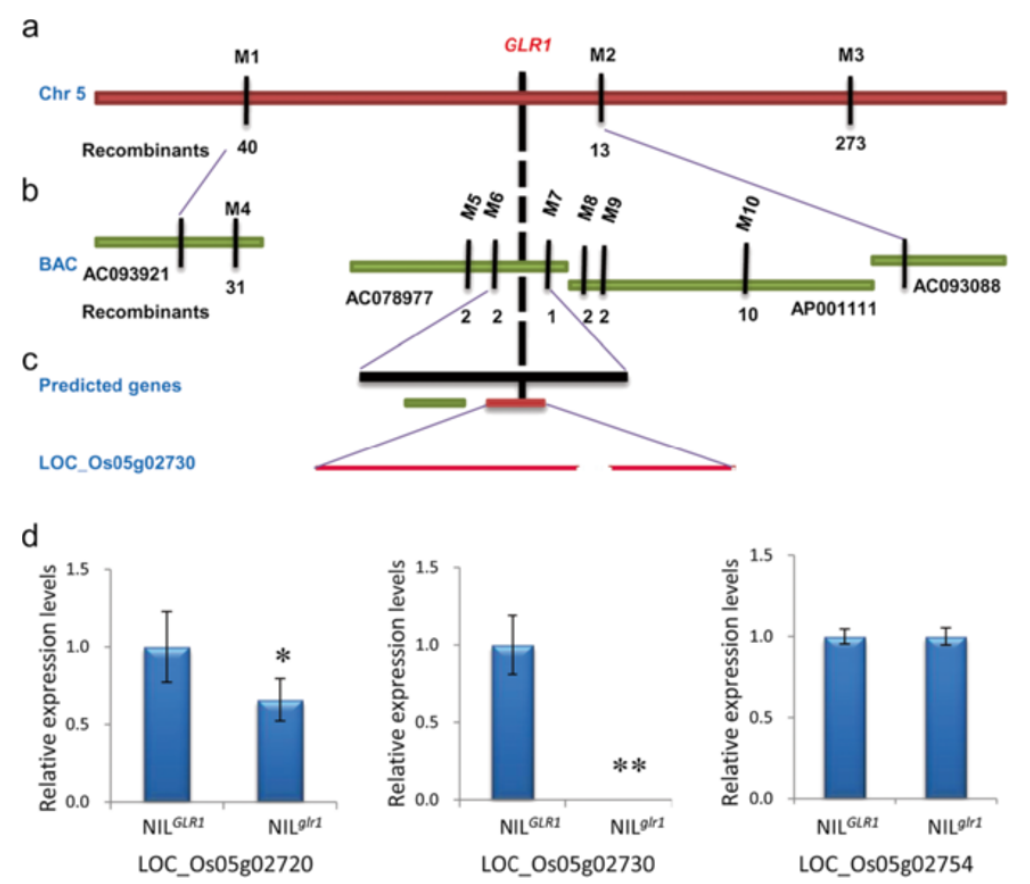

Figure 2 Map-based cloning of GLR1. (a) The GLR1 locus was mapped in chromosome 5 between markers M1 and M2. Recombinants were identified from 1,447 $\mathrm{F}_{2}$ glabrous plants. (b) Fine mapping of the GLR1 locus. The GLR1 locus was narrowed to a 21-kb genomic DNA region between markers M6 and M7. (c) The LOC_Os05902720 (green) and LOC_Os05g02730 (red) are predicted in the candidate region. The annotated gene of LOC_Os05g02730 consists of two exons and one intron. (d) The relative expression levels of LOC_Os05g02720, LOC_Os05g02730 and LOC_Os05g02754 in young panicles of NIL ${ }^{G L R T}$ and NIL ${ }^{\text {Ilr }}$ plants (T-test, $\mathrm{P}<0.05$ ).

Table 1 Molecular markers developed in this study

\begin{tabular}{|c|c|c|c|c|}
\hline Primer & & Primer sequence(5/-3/) & Primer types & Genetic distance(cM) \\
\hline \multirow[t]{2}{*}{ M1 } & Forward & TGGTTATTGTTATTTAGTTGGGTG & InDell & 1.38 \\
\hline & Reverse & TAGACTAGAGTTGGAGACG & & \\
\hline \multirow[t]{2}{*}{ M2 } & Forward & ACGCACGCCATTACAAAC & SSR & 0.44 \\
\hline & Reverse & CAGGAGGTGGGCCTCATT & & \\
\hline \multirow[t]{2}{*}{ M3 } & Forward & ACGACCCACCAGCAGATA & InDell & 9.43 \\
\hline & Reverse & AGGGACGTGAATGAAACT & & \\
\hline \multirow[t]{2}{*}{ M4 } & Forward & GCCCTTGATCCGGTGCTCT & InDell & 1.07 \\
\hline & Reverse & GTGTTAGATGCGTGTATT & & \\
\hline \multirow[t]{2}{*}{ M5 } & Forward & GGGGAAGCTCATTGTCGG & InDell & 0.10 \\
\hline & Reverse & CAGTGGTGGAGTCAAAAT & & \\
\hline \multirow[t]{2}{*}{ M6 } & Forward & GTAGTAGTAGGAGCACAGC & InDell & 0.07 \\
\hline & Reverse & CAATGCTGCATGGTGGTA & & \\
\hline \multirow[t]{2}{*}{ M7 } & Forward & AACAAATCCTCCTGTTCC & CAPS & 0.07 \\
\hline & Reverse & CGAGCTACTACTCCTGCT & & \\
\hline \multirow[t]{2}{*}{ M8 } & Forward & ATTGCTGGCACATTTTCT & InDell & 0.07 \\
\hline & Reverse & CATITTCTTCCTATCTAA & & \\
\hline \multirow[t]{2}{*}{ M9 } & Forward & CTAAGCAAGCTGACGTGTAAT & InDell & 0.07 \\
\hline & Reverse & AACCAAATAGCACTITCACA & & \\
\hline \multirow[t]{2}{*}{ M10 } & Forward & TCTGTTTCGTTGGATTAGT & InDell & 0.04 \\
\hline & Reverse & ACGAGGCATTCTTGATGG & & \\
\hline
\end{tabular}


WOX genes are specifically expressed in the epidermal cells and play important roles in their differentiation (Dai, et al. 2007, Ishida, et al. 2008, Matsumoto and Okada 2001, Nardmann, et al. 2004, Vernoud et al. 2009). Therefore, LOC_Os05g02730 is most likely the candidate gene responsible for the rice glabrous phenotype.

\section{Altering the expression levels of GLR1 could partially change the glabrous phenotype}

To confirm LOC_Os05g02730 is the GLR1 gene, we generated transgenic plants in a pubescence japonica variety Nipponbare background by the RNA interference (RNAi) method (Figure 4a). SEM analysis showed that much fewer trichomes on leaves of the RNAi transgenic lines have been observed (Figure 4b), and a further statistical analysis showed that the macrohair number on the RNAi transgenic leaves was significantly decreased (Figure 4c). When constitutively express GLR1 in NIL ${ }^{g l r 1}$, it can partially rescue glabrous phenotype of NIL ${ }^{g l r 1}$ of T0 transgenic plants (Figure $4 \mathrm{~d}$-g). These results indicate that LOC_Os05g02730 is the gene responsible for the glabrous phenotype of the NIL ${ }^{g l r 1}$ plant.

\section{DNA methylation may be involved in the expression of GLR1}

The findings that no mutation was found in the GLR1containing mapping region and that the expression of $L O C_{-} O s 05 g 02730$ was unable to be detected in the $\mathrm{NIL}^{g l r} 1$ plant strongly suggests that GLR1 may be regulated epigenetically through a DNA methylation mechanism. We therefore carried out a bisulfite sequencing experiment to examine whether DNA methylation are involved in the regulation of GLR1. As shown in Figure 5, the bisulfite sequencing of the $2.0-\mathrm{kb}$ promoter region of GLR1 revealed some apparent methylation differences between $\mathrm{NIL}^{\text {glr } 1}$ and NIL ${ }^{G L R 1}$, suggesting that an epigenetic mechanism may involve in the regulation of the GLR1 expression.

\section{Discussion}

In Arabidopsis, trichomes have been served as an excellent model system to study plant cell differentiation (Ishida et al. 2008). Glabrous mutants that are defective in leaf hair or trichome have been identified in many plant species. However, genes controlling trichome development in rice have not been identified up to date yet. GL1 was previously mapped on the short arm of chromosome 5 ( $\mathrm{Li}$, et al. 2010, Wang et al. 2009, Yu et al. 1995) and it was proposed that a single nucleotide mutation (A to $\mathrm{T}$ ) in the 5'UTR of LOC_Os05g02754 (Os05g0118900), which encode an unknown protein, might be responsible for the $g l 1$ trait (Li, et al. 2010). Our mapping data suggested that $g l r 1$ may be allelic to $g l 1$. We compared the sequences of 5'UTR of LOC_Os05g02754 (Os05g0118900) of NIL ${ }^{G L R 1}$ and NIL ${ }^{g l r 1}$ and found that the indicated position of 5' UTR of LOC_Os05g02754 (Os05g0118900) in the NIL ${ }^{\text {GLR1 }}$ plant is $\mathrm{A}$ and that in the NIL ${ }^{\text {glr }}$ plant is T. However, our data indicate that instead of LOC_Os05g02754 (Os05g0118900) 

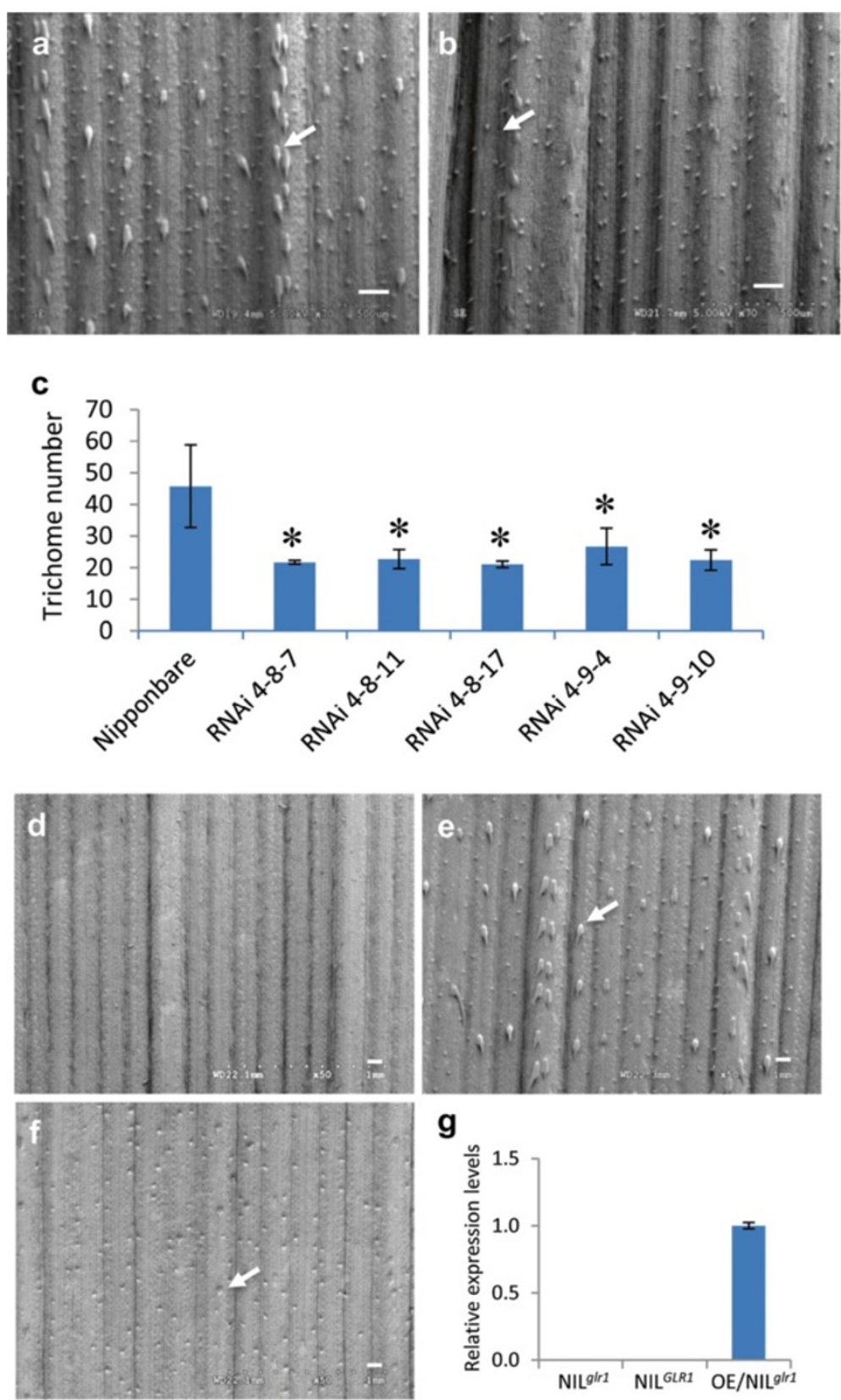

Figure 4 Glabrous phenotypes of GLR1 RNAi transgenic lines. (a, b) The SEM images of the abaxial leaf sides of Nipponbare (a) and GLR1 RNAi transgenic plants (b). (c) The macrohair number was significantly decreased in GLR1 RNAi transgenic plants compared with that in Nipponbare (T-test, $\mathrm{P}<0.05)$; (d-f) The SEM images of the abaxial leaf sides of NIL ${ }^{|l| r}(\mathbf{d})$, NIL ${ }^{G L R T}(\mathbf{e})$ and OE/NIL ${ }^{\text {glr } 1}$ transgenic plants $(\mathbf{f}) ;(\mathbf{g})$ The relative expression levels of LOC_Os05g02730 in leaves of NIL ${ }^{\text {glr }}$, NIL ${ }^{\text {GLR }}$ and OE/NIL ${ }^{\text {Ilr } 1}$ plants. Arrows indicate the macrohairs, bar $=1 \mathrm{~mm}$.

LOC_Os05g02730 (Os05g0118700), which encode a WUSlike homeodomain protein, controls the glabrous phenotype. First, the mapping data has pinpointed the GLR1 locus within a $21-\mathrm{kb}$ region that contains only two predicted genes, LOC_Os05g02720 (Os05g0118600) and LOC_Os05g02730 (Os05g0118700). Second, comparison of the gene expression levels of LOC_Os05g02720 and LOC_Os05g02730 between NIL ${ }^{\text {GLR1 }}$ and NIL ${ }^{\text {glr1 }}$ plants showed that LOC_Os05g02730 is dramatically increased in the NIL ${ }^{\text {glr } 1}$ plant, however, change of LOC_Os05g02720 in the NIL ${ }^{g l r 1}$ plant is not very significant. In contrast, no difference of expression levels of LOC_Os05g02754 has been detected between NIL ${ }^{G L R 1}$ and NIL ${ }^{g l r 1}$ plants (Figure 2d). Third, an apparent decrease in the trichome number on leaves and glumes of GLR1 RNAi transgenic plants have been obtained. Overexpression of GLR1 in NIL ${ }^{g l r}$ partially rescues glabrous phenotype of the NIL ${ }^{g l r 1}$ plant. Fourth, the sequence alignment showed that GLR1 has high 


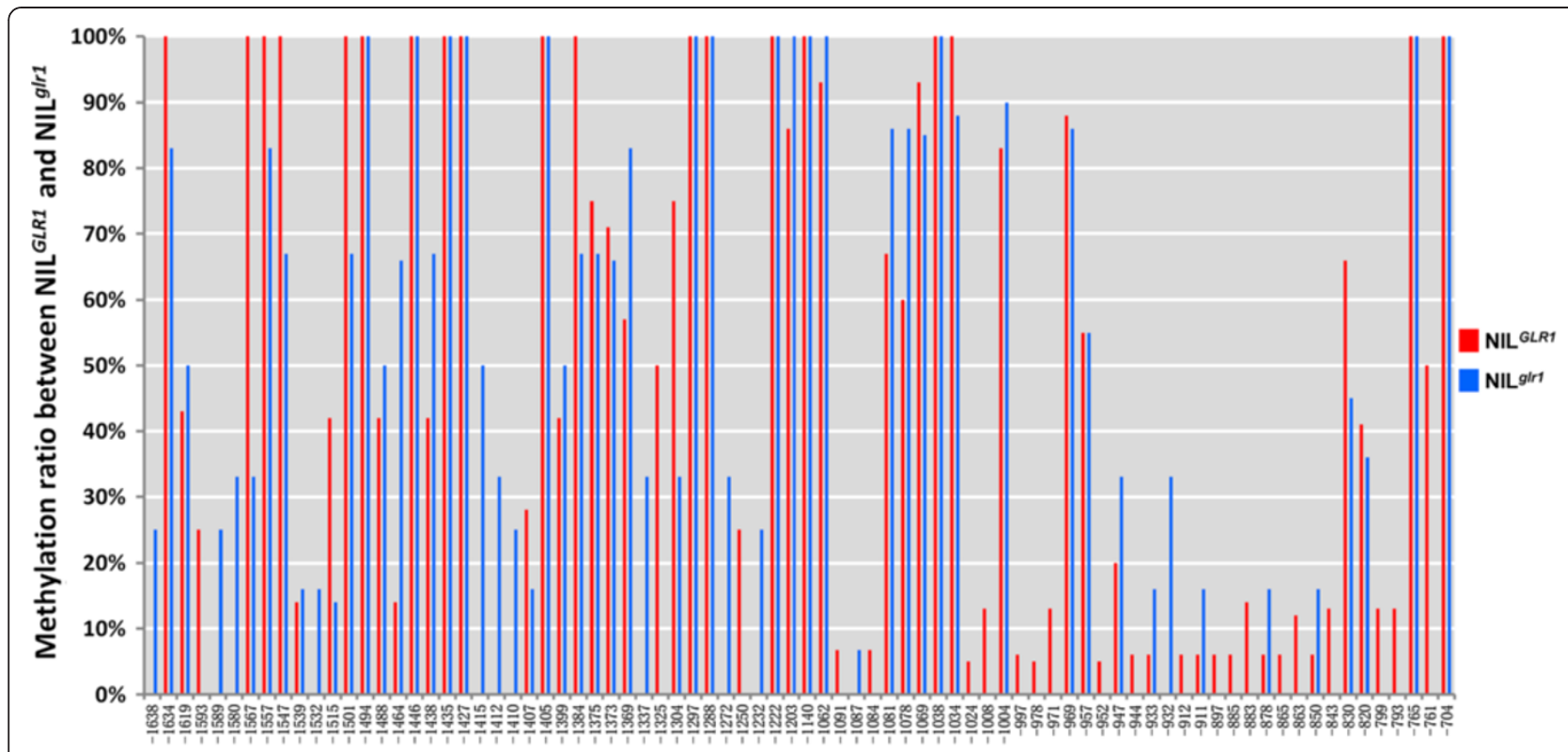

Figure 5 Comparison of the DNA methylation between NIL $^{\text {GLR1 }}$ and NIL $^{\text {glr1 }}$. DNA methylation levels (\%) of the GLR1 and glr1 DNA sequences of the NIL ${ }^{G L R 1}$ (red) and NIL ${ }^{g / r 1}$ (blue) plants are analyzed. The numbers indicate the position in the 2-kb upstream region starting from the start codon.

similarity to previously identified homeodomain proteins, whose functions are essential for the differentiation of epidermal cells (Dai, et al. 2007, Matsumoto and Okada 2001, Nardmann, et al. 2004). Taken all these together, we strongly suggest that the WUS-like homeodomain protein encoded by LOC_Os05g02730 is the GLR1 gene and is essential for the trichome development in rice.

The RNAi of GLR1 reduced the trichome number in transgenic lines, while the trichome is almost completely lost in NIL ${ }^{g l r l}$. This discrepancy could result from the different genetic background of plant materials or from incomplete suppression of the target gene in transgenic plants. Alternatively, the expression LOC_Os05g02720 is also decreased in the NIL ${ }^{\text {glr }}$ plant, which implies that LOC_Os05g02720 may be also involve in trichome development, thus knockdown of LOC_OsO5g02730 alone cannot completely suppress the trichome development. Further knockdown LOC_Os05g02720 along or knockdown it together with $L O C_{-}$OsO5g02730 will clarify the role of LOC_Os05g02720.

Chromatin state controls gene expression and plays critical roles in development. In plant, trimethylated $\mathrm{K} 9$ of histone H3 (H3K9me3) indicates an open chromatin state, while monomethylated and dimethylated H3K9s (H3K9me1 and H3K9me2) indicate a closed state (Liu et al. 2010). The identification of GL2 EXPRESSION MODULATOR (GEM) indicates that the regulation of GL2 expression is more complicated than previously expected (Caro, et al. 2007). Trichome density increased in gem-1 mutant whereas decreased in GEM-overexpressing plants.
Consistent with the phenotype, the GL2 expression has been observed to increase in gem-1 whereas decrease in GEM-overexpressing plants (Caro, et al. 2007). It has been observed that H3K9me3 increases and H3K9me2 decreases in the GL2 promoter in the gem-1 background, but H3K9me3 decreases and H3K9me2 increases in GEMoverexpressing plants (Caro, et al. 2007). This kind of epigenetic control may also be involved in rice trichome development. Rice SET Domain Group Protein 714 (SDG714) functions as a histone H3K9 methyltransferase, which is involved in histone H3K9 methylation, DNA methylation and genome stability (Ding et al. 2007). Loss of macrohairs but not microhairs on leaves of the SDG714 RNAi transgenic plants indicated that regulation of chromatin status of some unidentified regulators may play an important role in the trichome development in rice (Ding et al. 2007). In agreeable to these findings, the genomic bisulfite sequencing of GLR1 showed that the DNA methylation pattern at several sites of the GLR1 promoter region in the $\mathrm{NIL}^{\text {glr } 1}$ plants is different from that in the NIL ${ }^{\text {GLR } 1}$ plants, though no sequence difference of GLR1 was found between the NIL ${ }^{G L R ~} 1$ and NIL ${ }^{g l r 1}$ plants. These results indicated that the epigenetic mechanism may be involved in the regulation of the GLR1 expression and the trichome development in rice. Although, different patterns of the DNA methylation in upstream region of LOC_Os05g02730 $(O s 05 g 0118700)$ between $\mathrm{NIL}^{G L R 1}$ and $\mathrm{NIL}^{{ }^{g l r} 1}$ has been observed, we are unable to determine which sites are responsible for suppression of LOC_Os05g02730. Moreover, the GLR1 expression driven by a constitutive promoter 
dramatically increased the expression of GLR1, but cannot completely rescue the glabrous phenotype in T0 transgenic plants (Figure $4 \mathrm{~d}$-g). It indicates that the regulation of the GLR1 expression and the trichome development in rice is more complicate than expected. Further investigation is needed to uncover the molecular mechanism of GLR1 expression regulation.

Glabrous rice varieties are widely cultivated in America and Africa, while most varieties cultivated in Asia are pubescent (Khush, et al. 2001). In higher plants, although trichomes are thought to be important for plant defense against biotic and abiotic stresses, glabrous trait may be a selectively neutral trait in rice. Previous studies have indicated that the introduction of glabrous trait into japonica varieties may not cause any obvious disadvantages in plant defense (Li et al. 2011). In agriculture, the interest of breeding glabrous elite rice varieties is mainly due to its practical advantages of greater packing capability and less itching effect during the harvest process. The cloning of GLR1 will not only help to understand the molecular mechanism of trichome development in rice but also improve the efficiency of breeding glabrous elite rice varieties by marker-assisted selection and genetic modification approaches.

\section{Conclusions}

GLR1 plays an important role in rice trichome development and will contribute to breeding of glabrous elite rice varieties

\section{Methods}

\section{Plant materials}

Jia64 is a glabrous variety derived from American rice variety Rico No.1 and Jia33 is a pubescent variety in southeast China. Rice plants were cultivated in the experimental field of Jiaxing Academy of Agricultural Science in growing seasons from May to October.

\section{Scanning electron microscopy}

Samples were prepared as described previously ( $\mathrm{Li}$ et al. 2009). Briefly, samples were fixed with $2.5 \%$ (v/v) glutaraldehyde in $0.1 \mathrm{M}$ sodium phosphate buffer (PBS, pH 7.2) at $4^{\circ} \mathrm{C}$ overnight. After being rinsed with $0.1 \mathrm{M}$ PBS twice, samples were post-fixed in $1 \%(\mathrm{w} / \mathrm{v})$ osmium tetroxide for $2 \mathrm{~h}$ at $4^{\circ} \mathrm{C}$. Samples were rinsed with the same buffer for 2 more times and then dehydrated in a graded series of ethanol. For scanning electron microscopy, samples were critical-point dried (Hitachi HCP-2) and observed under a scanning electron microscope (Hitachi S-3000N).

\section{Genetic mapping of GLR1}

An $F_{2}$ mapping population was generated from a cross between Jia64 and Jia33. 24 molecular markers were used for genetic linkage analysis of $44 \mathrm{~F}_{2}$ plants that show the glabrous phenotype. To fine-map GLR1, new PCR-based markers were developed and 1,447 $\mathrm{F}_{2}$ glabrous plants were analyzed using markers as given in Table 1. The GLR1 locus was further narrowed within an interval of 21-kb DNA fragment between the M6 and M7 markers. To sequence the GLR1 locus, the entire genomic region was amplified from NIL ${ }^{G L R 1}$ and NIL ${ }^{\text {glr } 1}$ by PCR with LA-Taq (TaKaRa).

\section{RNA extraction and reverse transcription-polymerase chain reaction ( $R T-P C R$ )}

Total RNA was isolated from rice plants by Trizol extraction method (Invitrogen Life Technologies). To conduct RT-PCR analyses, cDNA strands are synthesized by the SuperScript III RT kit (Invitrogen Life Technologies). Real-time PCR analysis were performed using the SYBR Green RT-PCR kit (Biorad). Primers RT1-F and RT1-R were used to amplify LOC_Os05g02720, RT2-F and RT2$\mathrm{R}$ to LOC_Os05g02730 and primers RT3-F and RT3-R to LOC_Os05g02754 (Table 2).

\section{Plasmid construction and rice transformation}

To generate the RNAi construct, two DNA fragments RNAi 1-1 and RNAi 1-2 were amplified respectively by primers RNAi 1-1f and RNAi1-1r, RNAi1-2f and RNAi12r (Table 2). The construct 1460-RNAi 1-1 was generated by digesting the RNAi 1-1 fragment with BamH I and Kpn I and ligated to the binary vector 1460 by T4 DNA ligases. The hairpin cassette was generated by digesting the RNAi 1-2 fragment with Sac I and Spe I and ligated in reversed direction of fragment RNAi 1-1 to construct 1460-RNAi 1-1. For construction of the overexpression cassette, the coding region of GLR1 was amplified and liagated to the 1460 vector by BamH I and Spe I. The constructs were confirmed by sequencing and introduced into Agrobacterium tumefaciens strain EHA105 by electroporation. The rice (Nipponbare)

\section{Table 2 Primers for RT-PCR and RNAi construct}

\begin{tabular}{ll}
\hline Primer & Primer sequence (5/-3/) \\
\hline RT1-F & GGCAAGGCATCAGTTAGTG \\
RT1-R & GCCAGAGGTCCTCCAA \\
RT2-F & GCCGCAGCAGCAGCAGCAGCAGCTTACA \\
RT2-R & TCCACTAGCTTCCCCAGCGAGTAGTCCG \\
RT3-F & GTCCTCCCTCAGCTTCTCATCGTCA \\
RT3-R & GAAGCACATCGCCGCCGTCTCC \\
RNAi1-1f & ACGGATCCTTAATCATTGCTAATCGATCA \\
RNAi1-1r & GAGGTACCCGTCATGCTGCTCTTCCT \\
RNAi1-2f & GGAGCTCTTAATCATTGCTTAATCGATCA \\
RNAi1-2r & GCACTAGTCGTCATGCTGCTCTTCCT \\
\hline
\end{tabular}


Table 3 Primers for Bisulfite sequencing

\begin{tabular}{|c|c|}
\hline Primer & Primer sequence(5/-3/) \\
\hline ME-3F & AGAGTTGTTATGGGTGTAATGTTGTTTA \\
\hline MEQ-3F & GATTTGTTATTATAGGTATTATAAAGAGA \\
\hline$M E-3 R$ & TTACATCTCATAAAAATATTTTATTAACT \\
\hline MEQ-3R & TAAAAAACAAACACCTATCCCTATCCTAC \\
\hline$M E-4 F$ & TाTTGAGAATTATTAGATTIITTATGGT \\
\hline MEQ-4F & TTGTITITATTAATTATTTATTAAT \\
\hline$M E-4 R$ & AATACTAATAAACAATACATCAATCCTCTT \\
\hline MEQ-4R & CCACAATAAAACATAAAAATCACAAAACTA \\
\hline$M E-5 F$ & AATATAATGGATTATTTGGTGGATTAGTTT \\
\hline MEQ-5F & GGTAATTTTTITTTTATTTTTAGTGTTA \\
\hline$M E-5 R$ & ACATAACACACTAAAACAAAAAATTCATA \\
\hline MEQ-5R & CTITACATCATCACTATATAATAACAATT \\
\hline ME-6F & GGTTATTTGGATTATGTTAATATGTTAGG \\
\hline MEQ-6F & TITAAGTTGGTAGTIIITITGGTIITAG \\
\hline ME-7F & ATATTATATTAGATGTGGGAGTATTAATT \\
\hline MEQ-7F & AGGTTATTTGGATTATGTTAATATGTTAG \\
\hline ME-7R & ATATAACTATTATTTAATTAATACCTAACT \\
\hline MEQ-7R & TAAATATAATTACTTCCAATCAATTAAA \\
\hline ME-8F & TTGAATAAAATATGTAGTAATATGTTTATT \\
\hline MEQ-8F & TTGTATATTTGGGGTGGTAATTTATT \\
\hline ME-8R & ATCAACCCCACCACCACCACCCCCATCAA \\
\hline MEQ-8R & TCCACCACCACCACCACTCTACTACTACTA \\
\hline ME-9F & ATATAAGAAAATTTAGTTATTTAGGTAG \\
\hline MEQ-9F & ATAGGAGGAGGGATATATGGTGTTGGTGGT \\
\hline ME-9R & AAAAATAATAAAAAATACAAAAACAACAA \\
\hline MEQ-9R & CAAAACTTCTAACAATCACAAACCTTATA \\
\hline
\end{tabular}

transformation was performed as described previously (Hiei et al. 1994). For RNAi transgenic plants, T2 lines derived from individual transgenic lines were used for further analysis. T2 Lines RNAi 4-8-7, RNAi 4-8-11 and RNAi 4-8-17 were derived from line RNAi 4-8. T2 Lines RNAi 4-9-4 and RNAi 4-9-11 were derived from line RNAi 4-9. For overexpression transgenic plants, T0 plants were used for analysis.

\section{Bisulfite sequencing}

Genomic DNA extracted by the CTAB method and $1.0 \mu \mathrm{g}$ DNA was bisulfite treated using the Bisulfite kit (Qiagen 59104). The candidate $2-\mathrm{kb}$ upstream of the coding region of LOC_Os05g02730 was amplified using listed bisulfite primers (Table 3 ). The PCR products were cloned into the pGEM-T easy vector (Promega) for sequencing.

\section{Competing interests}

The authors declare that they have no competing interests.

\section{Authors' contributions}

$J L$ and $Y Y$ preformed genetic analyses and cloned the GLR1 gene, $Y Y, Z L, L Y$, $R G$, JL and GX conducted functional characterization. JL, GX conceived the proposal. GX wrote the manuscript. JL correct the final manuscript. All authors read and approved the final manuscript.

\section{Acknowledgements}

This work is supported by grants (2010C12002 and 011102471) from Department of Science and Technology of Zhejiang Province.

\section{Author details}

1 Jiaxing Academy of Agricultural Sciences, Jiaxing, Zhejiang Province 314016, China. ${ }^{2}$ State Key Laboratory of Plant Genomics and National Center for Plant Gene Research, Institute of Genetics and Developmental Biology, Chinese Academy of Sciences, Beijing 100101, China.

Received: 30 March 2012 Accepted: 27 September 2012 Published: 6 October 2012

\section{References}

Caro E, Castellano MM, Gutierrez C (2007) A chromatin link that couples cell division to root epidermis patterning in Arabidopsis. Nature 447:213-217

Dai M, Hu Y, Zhao Y, Liu H, Zhou DX (2007) A WUSCHEL-LIKE HOMEOBOX gene represses a $Y A B B Y$ gene expression required for rice leaf development. Plant Physiol 144:380-390

Ding Y, Wang X, Su L, Zhai J, Cao S, Zhang D, Liu C, Bi Y, Qian Q, Cheng Z, Chu C, Cao X (2007) SDG714, a histone H3K9 methyltransferase, is involved in Tos 17 DNA methylation and transposition in rice. Plant Cell 19:9-22

Gan Y, Kumimoto R, Liu C, Ratcliffe O, Yu H, Broun P (2006) GLABROUS INFLORESCENCE STEMS modulates the regulation by gibberellins of epidermal differentiation and shoot maturation in Arabidopsis. Plant Cell 18:1383-1395

Gan Y, Liu C, Yu H, Broun P (2007a) Integration of cytokinin and gibberellin signalling by Arabidopsis transcription factors GIS, ZFP8 and GIS2 in the regulation of epidermal cell fate. Development 134:2073-2081

Gan Y, Yu H, Peng J, Broun P (2007b) Genetic and molecular regulation by DELLA proteins of trichome development in Arabidopsis. Plant Physiol 145:1031-1042

Guo L, Luo L, Zhong D, Yu X, Mei H, Wang Y, Ying C (1999) Evaluation, improvement and utilization on some selected American rice cultivars. J Zhejiang Agric Sci 5:201-206

Hiei Y, Ohta S, Komari T, Kumashiro T (1994) Efficient transformation of rice (Oryza sativa L.) mediated by Agrobacterium and sequence analysis of the boundaries of the T-DNA. Plant J 6:271-282

Ishida T, Kurata T, Okada K, Wada T (2008) A genetic regulatory network in the development of trichomes and root hairs. Annu Rev Plant Biol 59:365-386

Khush GS, Brar DS, Hardy B (2001) Rice genetics IV. Proceedings of the Fourth International Rice Genetics Symposium, 22-27 October 2000, Los Baños, Philippines. Science Publishers, Inc, Enfield, NH (USA), p 29, Los Baños (Philippines): International Rice Research Institute

Lauter N, Gustus C, Westerbergh A, Doebley J (2004) The inheritance and evolution of leaf pigmentation and pubescence in teosinte. Genetics 167:1949-1959

Li J, Lin Z, Chen H, Xu M (1993) The inheritance of glabrous-leaf character in rice variety Rico No. 1. Acta Agriculturae Zhejiangensis 5:233-234

Li JJ, Tao R, Shi J, Hao Z, Gao R, Li L (2011) Resistance identification of the rice varieties in southeastern China to rice stripe disease. Acta Phytophylacica Sinica 38:221-226

Li S, Qian Q, Fu Z, Zeng D, Meng X, Kyozuka J, Maekawa M, Zhu X, Zhang J, Li J, Wang Y (2009) Short paniclel encodes a putative PTR family transporter and determines rice panicle size. Plant J 58:592-605

Li W, Wu J, Weng S, Zhang D, Zhang Y, Shi C (2010) Characterization and fine mapping of the glabrous leaf and hull mutants ( $g / 1)$ in rice (Oryza sativa L.). Plant Cell Rep 29:617-627

Liu C, Lu F, Cui X, Cao X (2010) Histone methylation in higher plants. Annu Rev Plant Biol 61:395-420

Luo J, Hu P, Tang S, Fasong H (2000) American glabrous rice utilized in breeding of super-high-yielding and good-quality varieties. Chin Rice Res Newsl 8:3-5

Machado A, Wu Y, Yang Y, Llewellyn DJ, Dennis ES (2009) The MYB transcription factor GhMYB25 regulates early fibre and trichome development. Plant J 59:52-62 
Maes L, Inze D, Goossens A (2008) Functional specialization of the TRANSPARENT TESTA GLABRA1 network allows differential hormonal control of laminal and marginal trichome initiation in Arabidopsis rosette leaves. Plant Physiol 148:1453-1464

Matsumoto N, Okada K (2001) A homeobox gene, PRESSED FLOWER, regulates lateral axis-dependent development of Arabidopsis flowers. Genes Dev 15:3355-3364

Moose SP, Lauter N, Carlson SR (2004) The maize macrohairless 1 locus specifically promotes leaf blade macrohair initiation and responds to factors regulating leaf identity. Genetics 166:1451-1461

Nagao S, Takahashi M-E, Kinoshita T (1960) Genetical studies on rice plant, XXV: inheritance of threemorphological characters, pubescence of leaves and floral glumes, and deformation of empty glumes. Journal of the Faculty of Agriculture, Hokkaido University, pp 299-314

Nardmann J, Ji J, Werr W, Scanlon MJ (2004) The maize duplicate genes narrow sheath1 and narrow sheath2 encode a conserved homeobox gene function in a lateral domain of shoot apical meristems. Development 131:2827-2839

Perazza D, Vachon G, Herzog M (1998) Gibberellins promote trichome formation by up-regulating GLABROUS1 in Arabidopsis. Plant Physiol 117:375-383

Qi T, Song S, Ren Q, Wu D, Huang H, Chen Y, Fan M, Peng W, Ren C, Xie D (2011) The Jasmonate-ZIM-domain proteins interact with the WD-Repeat/ bHLH/MYB complexes to regulate Jasmonate-mediated anthocyanin accumulation and trichome initiation in Arabidopsis thaliana. Plant Cell 23:1795-1814

Rerie WG, Feldmann KA, Marks MD (1994) The GLABRA2 gene encodes a homeo domain protein required for normal trichome development in Arabidopsis. Genes Dev 8:1388-1399

Schellmann S, Kirik SA, V Wada T, Okada K, Beermann A, Thumfahrt J, Jurgens G, Hulskamp M (2002) TRIPTYCHON and CAPRICE mediate lateral inhibition during trichome and root hair patterning in Arabidopsis. EMBO J 21:5036-5046

Traw MB, Bergelson J (2003) Interactive effects of jasmonic acid, salicylic acid, and gibberellin on induction of trichomes in Arabidopsis. Plant Physiol 133:1367-1375

Vernoud V, Laigle G, Rozier F, Meeley RB, Perez P, Rogowsky PM (2009) The HD-ZIP IV transcription factor OCL4 is necessary for trichome patterning and anther development in maize. Plant J 59:883-894

Wang D, Sun S-X, Gao F-Y, Lu X-J, Li Z-H, Ren G-J (2009) Mapping a rice Glabrous gene using simple sequence repeat markers. Rice Sci 16:93-98

Yang C, Li H, Zhang J, Luo Z, Gong P, Zhang C, Li J, Wang T, Zhang Y, Lu Y, Ye Z (2011) A regulatory gene induces trichome formation and embryo lethality in tomato. Proc Natl Acad Sci USA 108:11836-11841

Yu N, Cai WJ, Wang S, Shan CM, Wang LJ, Chen XY (2010) Temporal control of trichome distribution by microRNA156-targeted SPL genes in Arabidopsis thaliana. Plant Cell 22:2322-2335

Yu ZH, McCouch SR, Tanksley SD, Kinoshita T, Sato S (1995) Association of morphological and RFLP markers in rice (Oryza sativa L.). Genome 38:566-574

Zhou Z, An L, Sun L, Zhu S, Xi W, Broun P, Yu H, Gan Y (2011) Zinc finger protein 5 is required for the control of trichome initiation by acting upstream of zinc finger protein 8 in Arabidopsis. Plant Physiol 157:673-682

doi:10.1186/1939-8433-5-32

Cite this article as: Li et al.: Glabrous Rice 1, encoding a homeodomain protein, regulates trichome development in rice. Rice 2012 5:32.

\section{Submit your manuscript to a SpringerOpen ${ }^{\circ}$ journal and benefit from:}

- Convenient online submission

- Rigorous peer review

- Immediate publication on acceptance

- Open access: articles freely available online

- High visibility within the field

- Retaining the copyright to your article

Submit your next manuscript at $>$ springeropen.com 